\title{
Five-year results of a randomised comparison of titanium- nitride-oxide-coated stents with zotarolimus-eluting stents for coronary revascularisation
}

Thomas Pilgrim ${ }^{1}, \mathrm{MD}$; Lorenz Räber ${ }^{1}, \mathrm{MD}$; Andreas Limacher ${ }^{2}, \mathrm{PhD}$; Peter Wenaweser ${ }^{1}, \mathrm{MD}$;

Stéphane Cook ${ }^{3}$, MD; Jean-Christophe Stauffer ${ }^{3}$, MD; Ali Garachemani ${ }^{4}$, MD; Aris Moschovitis ${ }^{1}$, MD;

Bernhard Meier ${ }^{1}$, MD; Peter Jüni ${ }^{2,5}$, MD; Stephan Windecker ${ }^{1,2 *}$, MD

1. Department of Cardiology, Swiss Cardiovascular Center, Bern University Hospital, Bern, Switzerland; 2. Clinical Trials Unit Bern, Department of Clinical Research, University of Bern, Bern, Switzerland; 3. Cardiology, University of Fribourg, Fribourg, Switzerland; 4. Lindenhof Hospital, Bern, Switzerland; 5. Institute of Social and Preventive Medicine, University of Bern, Bern, Switzerland

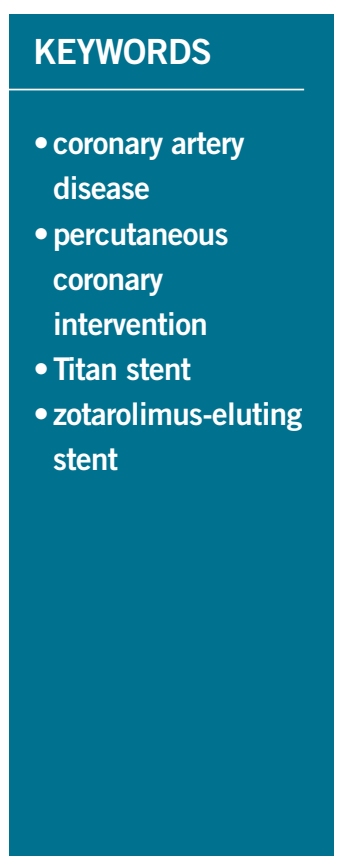

\begin{abstract}
Aims: Stents with a passive coating of titanium-nitride-oxide (TiNO) have been compared with Endeavor ${ }^{\mathrm{B}}$ zotarolimus-eluting stents (E-ZES) with regard to the primary endpoint of in-stent late lumen loss at six to eight months. The objective of the present analysis was to compare the long-term outcomes of TiNO stents with E-ZES up to five years of clinical follow-up.

Methods and results: A total of 302 patients had been randomly allocated to treatment with TiNO or E-ZES. Up to five years of follow-up, major adverse cardiac events (MACE), the composite of cardiac death, myocardial infarction, or clinically indicated target vessel revascularisation (TLR), were observed in $27.6 \%$ of patients treated with TiNO stents and $25.3 \%$ of patients treated with E-ZES (RR 1.13, 95\% CI: 0.72-1.75, $\mathrm{p}=0.60$ ), with the majority of events related to clinically indicated TVR (TiNO $21.7 \%$ versus E-ZES $20.7 \%$, RR $1.10,95 \%$ CI: $0.67-1.81$ ). There were no differences with respect to individual events including cardiac death, myocardial infarction or stent thrombosis between the two treatment arms up to five years of followup. A majority of patients remained free from angina throughout the entire study duration (TiNO $77.3 \%$ versus E-ZES $76.1 \%$, $\mathrm{p}=0.92$ ).
\end{abstract}

Conclusions: Final five-year outcomes of the TIDE trial comparing TiNO stents with E-ZES revealed increased rates of MACE driven primarily by clinically indicated TVR. The TIDE trial is registered at ClinicalTrials.gov: NCT00492908.

\footnotetext{
*Corresponding author: Department of Cardiology, Bern University Hospital, 3010 Bern, Switzerland. E-mail: stephan.windecker@insel.ch
} 


\section{Introduction}

Titanium-nitride-oxide-coated (TiNO) stents have been introduced to attenuate the acute inflammatory response to stent-mediated arterial injury and promote arterial healing while maintaining the antirestenotic efficacy. Passive stent coating with TiNO has been shown to decrease platelet adhesion and fibrinogen binding in vitro and to reduce neointimal hyperplasia in a porcine restenosis mode $^{1}$. In a randomised controlled trial, TiNO stents were superior to bare metal stents with regard to late luminal loss at six months and major adverse cardiac events (MACE) at six months and five years $^{2,3}$. TiNO stents were associated with a lower cumulative rate of MACE at five years of follow-up as compared with paclitaxeleluting stents (PES) in a randomised controlled trial of patients with acute myocardial infarction ${ }^{4}$.

In the TIDE trial, powered for a primary angiographic endpoint, TiNO stents were inferior to Endeavor ${ }^{\circledR}$ zotarolimus-eluting stents (E-ZES; Medtronic, Minneapolis, MN, USA) with regard to late loss and binary restenosis ${ }^{5}$. In the present analysis we present the final five-year clinical outcomes of the TIDE trial.

\section{Methods \\ PATIENT POPULATION}

Inclusion criteria of the TIDE trial have been published previously ${ }^{5}$. In summary, patients aged $\geq 18$ years presenting with stable angina or unstable angina with at least one lesion with a diameter stenosis of $\geq 50 \%$ were eligible for enrolment. All patients gave written informed consent for participation in the study, which was approved by the local ethics committee. The TIDE trial was an investigatorinitiated study supported by the Inselspital Foundation and performed without industry involvement. The study was registered at ClinicalTrials.gov: NCT00492908.

\section{STUDY DESIGN AND PROCEDURES}

The design and conduct of the TIDE study, an assessor-blind noninferiority trial with a primary angiographic endpoint at six to eight months, have been outlined previously ${ }^{5}$. Patients were randomly assigned to treatment with TiNO stents (Helistent Titan 2; Hexacath, Rueil-Malmaison, France) or E-ZES in a 1:1 fashion in three institutions in Switzerland.

\section{STUDY ENDPOINTS AND DEFINITIONS}

The primary endpoint of the study was in-stent late lumen loss at six to eight months after stent implantation as assessed by quantitative coronary angiography and was published previously.

MACE were defined as the composite of cardiac death, myocardial infarction or clinically indicated target vessel revascularisation (TVR). Follow-up was performed by the use of a standardised telephone interview yearly up to five years after intervention.

\section{STATISTICAL ANALYSIS}

Baseline clinical characteristics are reported as means and standard deviations and numbers and percentages as appropriate. Cumulative incidences of MACE and clinically indicated target lesion revascularisation (TLR) up to five years are shown by the Kaplan-Meier technique. We used the Mantel-Cox model and the corresponding log-rank test for between-group comparison of clinical outcomes occurring up to five years. All patients who underwent randomisation were included in the analysis in the group to which they were originally allocated (intention-to-treat principle). No adjustments were made for multiple comparisons; all p-values and 95\% confidence intervals are two-sided. All analyses were carried out using Stata Version 13 (StataCorp, College Station, TX, USA).

\section{Results}

A total of 302 patients had been randomly assigned to treatment with TiNO stents or E-ZES. Baseline clinical characteristics of the two treatment arms were comparable.

Clinical events at five years are summarised in Table 1. There was no difference in the rate of MACE between the two groups at five years (TiNO 27.6\% vs. E-ZES 25.3\%, RR 1.13, 95\% CI: 0.72-1.75, $\mathrm{p}=0.60$ ). The majority of MACE up to five years occurred within the first year of follow-up and were related to clinically indicated TVR in a majority of cases (Table 1, Figure 1), driven at least in part by protocol-mandated angiographic follow-up. Late events between one and five years were attributable to all-cause mortality, and clinically indicated TVR. TLR accounted for the majority of cases with clinically indicated TVR and is shown up to five years in Figure 2. One case of definite early stent thrombosis occurred in one of the

Table 1. Clinical outcome at 5 years.

\begin{tabular}{|c|c|c|c|c|}
\hline & $\begin{array}{c}\text { TNNO } \\
\text { (N=152) } \\
n(\%)\end{array}$ & $\begin{array}{c}\text { E-ZES } \\
(\mathrm{N}=150) \\
n(\%)\end{array}$ & $\begin{array}{l}\text { Mantel-Cox rate } \\
\text { ratio (RR) } \\
(95 \% \text { CI) }\end{array}$ & $\begin{array}{l}\text { log-rank } \\
p \text {-value }\end{array}$ \\
\hline Death & $13(8.6)$ & $8(5.3)$ & 1.63 (0.67 to 3.93$)$ & 0.28 \\
\hline Cardiac death & $5(3.3)$ & $4(2.7)$ & 1.25 (0.34 to 4.68$)$ & 0.74 \\
\hline Myocardial infarction & $8(5.3)$ & $11(7.3)$ & 0.71 (0.28 to 1.77$)$ & 0.46 \\
\hline Q-wave & $1(0.7)$ & $2(1.3)$ & $0.50(0.04$ to 5.50$)$ & 0.56 \\
\hline Non-Q-wave & $7(4.6)$ & $9(6.0)$ & 0.76 (0.28 to 2.04) & 0.58 \\
\hline Clinically indicated TLR & $24(15.8)$ & 19 (12.7) & 1.31 (0.71 to 2.40$)$ & 0.38 \\
\hline Percutaneous & $23(15.1)$ & $17(11.3)$ & 1.40 (0.74 to 2.63) & 0.29 \\
\hline Surgical & $2(1.3)$ & 2 (1.3) & 0.98 (0.14 to 6.98) & 0.98 \\
\hline Clinically indicated TVR & $33(21.7)$ & $31(20.7)$ & 1.10 (0.67 to 1.81$)$ & 0.70 \\
\hline Percutaneous & $32(21.1)$ & $28(18.7)$ & 1.19 (0.72 to 1.99 ) & 0.50 \\
\hline Surgical & $3(2.0)$ & $3(2.0)$ & 0.98 (0.20 to 4.86) & 0.98 \\
\hline Any repeat revascularisation & $51(33.6)$ & $41(27.3)$ & 1.32 (0.87 to 1.99$)$ & 0.19 \\
\hline Percutaneous & 49 (32.2) & $39(26.0)$ & 1.33 (0.87 to 2.03) & 0.19 \\
\hline Surgical & $4(2.6)$ & $4(2.7)$ & 0.99 (0.25 to 3.95$)$ & 0.98 \\
\hline Cardiac death or Ml & $12(7.9)$ & $14(9.3)$ & 0.84 (0.39 to 1.81 ) & 0.65 \\
\hline $\begin{array}{l}\text { Cardiac death, MI, or } \\
\text { clinically indicated TLR }\end{array}$ & $33(21.7)$ & 28 (18.7) & 1.20 (0.72 to 1.98$)$ & 0.49 \\
\hline $\begin{array}{l}\text { MACE (cardiac death, MI or } \\
\text { clinically indicated TVR) }\end{array}$ & $42(27.6)$ & $38(25.3)$ & $1.13(0.72$ to 1.75$)$ & 0.60 \\
\hline Stroke & $3(2.0)$ & $0(0.0)$ & $6.91(0.36 \text { to } 132.60)^{*}$ & $0.25^{*}$ \\
\hline
\end{tabular}

*Risk ratio with continuity correction of 0.5 and Fisher's exact test in case of zero events in one group. 


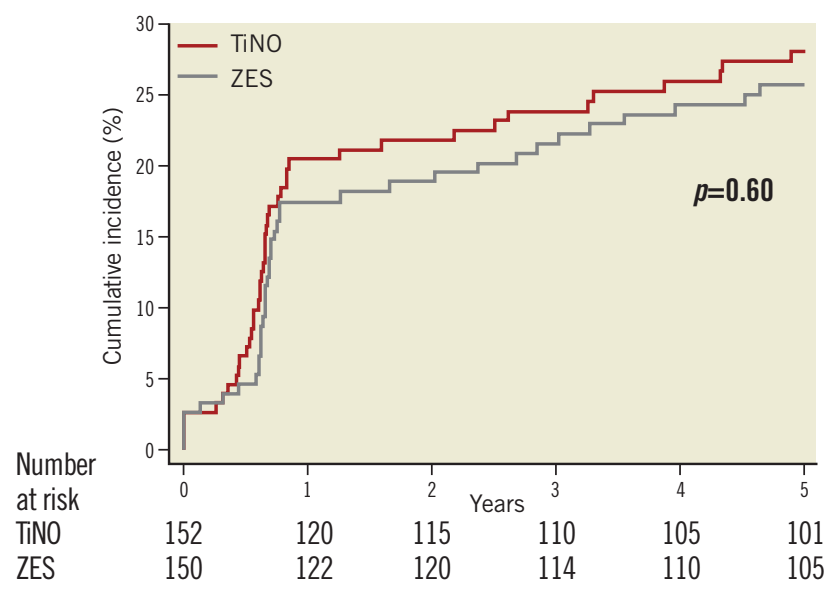

Figure 1. Major adverse cardiac events (composite of cardiac death, myocardial infarction or clinically indicated target vessel revascularisation). Cumulative event curves show the composite endpoint of cardiac death, myocardial infarction, and clinically indicated target vessel revascularisation up to five years of follow-up for TiNO stents and E-ZES.

patients treated with TiNO stents. There were no differences with respect to any of the individual outcomes between the two treatment arms up to five years of follow-up (Table 1). More than three quarters of the patients reported being free of angina at five years of follow-up (TiNO 102/152 [77.3\%] versus E-ZES 102/150 [76.1\%], $\mathrm{p}=0.89$ ). From $97 \%$ at year two, adherence to aspirin decreased to $92 \%$ and $95 \%$, respectively, at five years owing to more frequent use of clopidogrel (TiNO 11.9\% vs. E-ZES 7.3\%, p=0.22) (Table 2).

\section{Discussion}

The key messages of the final five-year clinical outcome of the randomised comparison between the TiNO stent and the E-ZES are the following. 1) There was no significant difference with regard to MACE between patients treated with the TiNO stent and the E-ZES

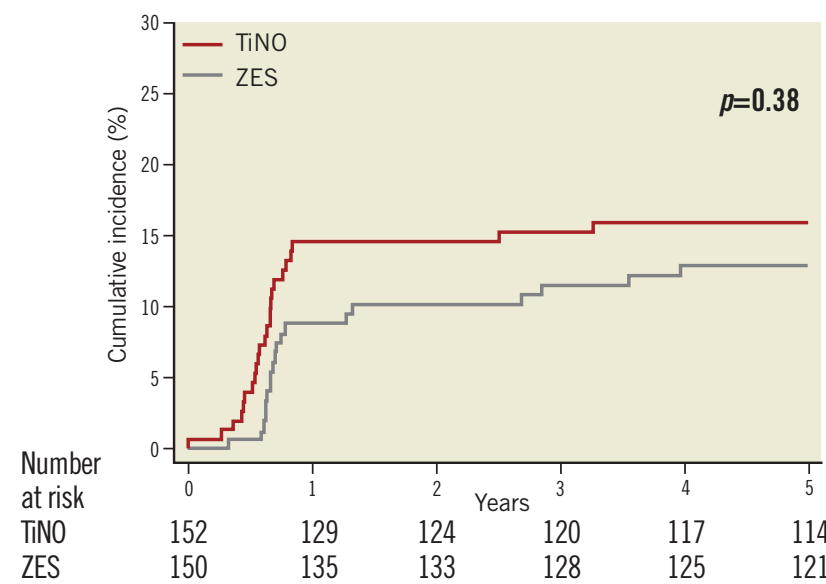

Figure 2. Clinically indicated target lesion revascularisation. Cumulative event curves show the clinically indicated target lesion revascularisations up to five years of follow-up for TiNO stents and E-ZES.
Table 2. Medications at 5 years.

\begin{tabular}{|l|c|c|c|}
\hline Aspirin & $\begin{array}{c}\text { TiNo } \\
(\mathbf{n = 1 3 5 )} \\
\mathbf{n}(\%)\end{array}$ & $\begin{array}{c}\text { ZES } \\
(\mathbf{n = 1 3 7 )} \\
\mathbf{n}(\%)\end{array}$ & $\begin{array}{c}\text { Fisher's } \\
\text { exact } \\
\boldsymbol{p} \text {-value }\end{array}$ \\
\hline Clopidogrel & $124(91.9)$ & $130(94.9)$ & 0.22 \\
\hline Oral anticoagulation & $16(11.9)$ & $10(7.3)$ & 0.22 \\
\hline Statin & $17(12.6)$ & $8(5.8)$ & 0.06 \\
\hline Other lipid-lowering drug & $111(82.2)$ & $120(87.6)$ & 0.24 \\
\hline ACE inhibitor & $56(41.5)$ & $4(2.9)$ & 0.68 \\
\hline ATII antagonist & $39(28.9)$ & $31(22.6)$ & 0.27 \\
\hline Beta-blocker & $84(62.2)$ & $88(64.2)$ & 0.80 \\
\hline Antiarrhythmic drug & $7(5.2)$ & $6(4.4)$ & 0.78 \\
\hline Ca antagonist & $36(26.7)$ & $29(21.2)$ & 0.32 \\
\hline Nitrates & $0(0.0)$ & $1(0.7)$ & 1.00 \\
\hline Diuretic & $36(26.7)$ & $25(18.2)$ & 0.11 \\
\hline Insulin & $10(7.4)$ & $8(5.8)$ & 0.63 \\
\hline Oral antidiabetic & $20(14.8)$ & $22(16.1)$ & 0.87 \\
\hline
\end{tabular}

up to five years of follow-up. 2) The majority of MACE in both treatment arms occurred within the first year after intervention and were frequently associated with clinically indicated TVR.

The present study has several limitations. First, the trial was powered for a primary angiographic endpoint and is hence underpowered to evaluate differences between TiNO and E-ZES with regard to clinical outcome. Second, protocol-mandated angiographic follow-up at six to eight months increased the rates of revascularisation procedures disproportionately. Third, both stent types have been superseded by novel stent technologies with improved safety and efficacy profiles. E-ZES has largely been replaced by the Endeavor Resolute DES (Medtronic), characterised by delayed and more sustained drug elution related to a novel polymer. However, the recent publication of the final results of the SORT OUT III trial emphasise the importance of long-term clinical outcomes in coronary stent trials to elucidate fully their safety and efficacy profiles ${ }^{6}$.

The rate of MACE up to five years was considerably higher in the TIDE trial $(27.6 \%)$ as compared to the event rates reported in the PORI registry $(16.9 \%)^{7}$ and even the TITAX AMI trial ${ }^{4}$. An "oculostenotic reflex" during the protocol-mandated repeat angiography may account for a difference in TLR, along with a high proportion of complex patients and longer lesion length compared to previous trials. The difference in clinical event rates between the TiNO stent and the E-ZES appeared to diminish during the course of the longterm follow-up.

The present trial was designed in the era of early-generation DES when long-term safety of DES had just been raised as a concern. With the introduction of newer-generation DES with high antirestenotic efficacy, improved strut endothelialisation, shortened minimal duration of dual antiplatelet therapy, and low rates of stent thrombosis, the role of passive stent coatings remains to be determined. Thin-strut DES releasing antiproliferative agents from 
biocompatible or biodegradable polymers demonstrated a rate of TLR ranging from $2 \%$ to $4 \%$ in recent trials ${ }^{8,9}$ and had negligible rates of very late $\mathrm{ST}$.

\section{Conclusion}

The final five-year outcomes of the TIDE trial comparing TiNO stents with E-ZES revealed increased rates of MACE driven primarily by clinically indicated TVR.

\section{Impact on daily practice}

Percutaneous coronary intervention with TiNO and E-ZES is associated with increased rates of MACE driven primarily by clinically indicated TVR. Both devices have been overtaken by newer iterations of drug-eluting stents.

\section{Conflict of interest statement}

T. Pilgrim has received lecture fees from Biotronik and Medtronic. P. Wenaweser has received a research grant to the institution from Medtronic, lecture and proctoring fees from Medtronic, Edwards, and Boston Scientific. P. Jüni is an unpaid steering committee or statistical executive committee member of trials funded by Abbott Vascular, Biosensors, Medtronic, and Johnson \& Johnson. CTU Bern, which is part of the University of Bern, has a staff policy of not accepting honoraria or consultancy fees. However, CTU Bern is involved in the design, conduct, or analysis of clinical studies funded by Abbott Vascular, Ablynx, Amgen, AstraZeneca, Biosensors, Biotronik, Boehringer Ingelheim, Eisai, Eli Lilly, Exelixis, Geron, Gilead Sciences, Nestle, Novartis, Novo Nordisc, Padma, Roche, Schering-Plough, St. Jude Medical, and Swiss Cardio Technologies. S. Windecker has received research contracts to the institution from Biotronik and St. Jude.

\section{References}

1. Windecker S, Mayer I, De Pasquale G, Maier W, Dirsch O, De Groot P, Wu YP, Noll G, Leskosek B, Meier B, Hess OM; Working Group on Novel Surface Coating of Biomedical Devices (SCOL). Stent coating with titanium-nitride-oxide for reduction of neointimal hyperplasia. Circulation. 2001;104:928-33.

2. Windecker S, Simon R, Lins M, Klauss V, Eberli FR, Roffi M, Pedrazzini G, Moccetti T, Wenaweser P, Togni M, Tüller D, Zbinden R, Seiler C, Mehilli J, Kastrati A, Meier B, Hess OM. Randomized comparison of a titanium-nitride-oxide-coated stent with a stainless steel stent for coronary revascularization: the TiNOX trial. Circulation. 2005;111:2617-22.
3. Moschovitis A, Simon R, Seidenstucker A, Klauss V, Baylacher M, Lüscher TF, Moccetti T, Windecker S, Meier B, Hess OM. Randomised comparison of titanium-nitride-oxide coated stents with bare metal stents: five year follow-up of the TiNOX trial. EuroIntervention. 2010;6:63-8.

4. Tuomainen PO, Ylitalo A, Niemela M, Kervinen K, Pietilä M, Sia J, Nyman K, Nammas W, Airaksinen KE, Karjalainen PP. Fiveyear clinical outcome of titanium-nitride-oxide-coated bioactive stents versus paclitaxel-eluting stents in patients with acute myocardial infarction: long-term follow-up from the TITAX AMI trial. Int J Cardiol. 2013;168:1214-9.

5. Pilgrim T, RaberL, LimacherA, LöffelL, WenaweserP, CookS, Stauffer JC, Togni M, Vogel R, Garachemani A, Moschovitis A, Khattab AA, Seiler C, Meier B, Jüni P, Windecker S. Comparison of titanium-nitride-oxide-coated stents with zotarolimus-eluting stents for coronary revascularization a randomized controlled trial. JACC Cardiovasc Interv. 2011;4:672-82.

6. Maeng M, Tilsted HH, Jensen LO, Krusell LR, Kaltoft A, Kelbæk H, Villadsen AB, Ravkilde J, Hansen KN, Christiansen EH, Aarøe J, Jensen JS, Kristensen SD, Bøtker HE, Thuesen L, Madsen M, Thayssen P, Sørensen HT, Lassen JF. Differential clinical outcomes after 1 year versus 5 years in a randomised comparison of zotarolimus-eluting and sirolimus-eluting coronary stents (the SORT OUT III study): a multicentre, open-label, randomised superiority trial. Lancet. 2014;383:2047-56.

7. Karjalainen PP, Ylitalo A, Airaksinen JK, Nammas W. Fiveyear clinical outcome of titanium-nitride-oxide-coated bioactive stent implantation in a real-world population: a comparison with paclitaxel-eluting stents: the PORI registry. J Interv Cardiol. 2011;24:1-8

8. Serruys PW, Silber S, Garg S, van Geuns RJ, Richardt G, Buszman PE, Kelbaek H, van Boven AJ, Hofma SH, Linke A, Klauss V, Wijns W, Macaya C, Garot P, DiMario C, Manoharan G, Kornowski R, Ischinger T, Bartorelli A, Ronden J, Bressers M, Gobbens P, Negoita M, van Leeuwen F, Windecker S. Comparison of zotarolimus-eluting and everolimus-eluting coronary stents. N Engl J Med. 2010;363:136-46.

9. von Birgelen C, Sen H, Lam MK, Danse PW, Jessurun GA, Hautvast RW, van Houwelingen GK, Schramm AR, Gin RM, Louwerenburg JW, de Man FH, Stoel MG, Löwik MM, Linssen GC, Saïd SA, Nienhuis MB, Verhorst PM, Basalus MW, Doggen CJ, Tandjung K. Third-generation zotarolimus-eluting and everolimuseluting stents in all-comer patients requiring a percutaneous coronary intervention (DUTCH PEERS): a randomised, single-blind, multicentre, non-inferiority trial. Lancet. 2014;383:413-23. 